\title{
Analysis of HOM Problems in the C-ADS Main Linac
}

\author{
Burn Huang ${ }^{1}$ and King Yuen $\mathrm{Ng}^{2}$
}

${ }^{1}$ Institute of Modern Physics, Chinese Academy of Sciences

509 Nanchang Road, Lanzhou, China 730000

${ }^{2}$ Fermi National Accelerator Laboratory

PO Box 500, Batavia, IL 60510, USA

(October, 2015)

(Finalized December, 2016)

\begin{abstract}
Excitation of higher-order modes (HOMs) in superconducting cavities may severely affect the operation of the main linac in the Chinese Accelerator Driven System (CADS)*. Preliminary analysis is made on the effects of beam dynamic, which includes possible longitudinal and transverse emittance enlargements, as well as the possibility of beam breakup. Suggestions are given for further investigation. Comparison is made between the C-ADS and the Fermilab Project X.
\end{abstract}

* Conceptual Physics Design on the $C$-ADS Accelerators, Ed. Jingyu Tang and Zhihui Li, Institute of High Energy Physics, CAS and Institute of Modern Physics, CAS, August 2012, IHEP-CADS-Report/2012-01E 


\title{
Analysis of HOM Problems in the C-ADS Main Linac
}

\author{
Burn Huang ${ }^{1}$ and King Yuen $\mathrm{Ng}^{2}$
}

${ }^{1}$ Institute of Modern Physics, Chinese Academy of Sciences 509 Nanchang Road, Lanzhou, China 730000

${ }^{2}$ Fermi National Accelerator Laboratory

PO Box 500, Batavia, IL 60510, USA

(October, 2015)

(Finalized December, 2016)

\begin{abstract}
Excitation of higher-order modes (HOMs) in superconducting cavities may severely affect the operation of the main linac in the Chinese Accelerator Driven System (CADS) [1]. Preliminary analysis is made on the effects of beam dynamic, which includes possible longitudinal and transverse emittance enlargements, as well as the possibility of beam breakup. Suggestions are given for further investigation. Comparison is made between the C-ADS and the Fermilab Project X.
\end{abstract}

\section{Introduction}

Superconducting rf (SRF) cavities are used in the China Accelerator Driven System (C-ADS) project [1]. SRF cavities have high quality factors, implying that the electromagnetic fields excited by a beam bunch do not decay much when successive bunches pass by. Besides the fundamental mode that is necessary for particle acceleration, unuseful higher-order modes (HOMs) will be excited as well. The accumulated HOM excited field energy can add to the cryogenic loss by so much that the maximum cryogenic heat load may be exceeded. The 
longitudinal HOM kicks experienced by the beam particles can enlarge the longitudinal bunch emittance, while the transverse HOM kicks can enlarge the transverse emittances. Excitation of HOMs can result in collective beam dynamic effects, one of which is coupled bunch instabilities. Another important one is successive accumulated transverse kicks that lead to beam breakup. For these reasons, the examination of HOM effects become an important issue to investigate when a linac is designed.

HOM couplers can be installed to extract HOM energy excited out of the cavities so as to avoid adverse HOM effects. Unfortunately, HOM couplers are expensive and may limit cavity performance and reduce operational reliability. HOM couplers were installed at the SNS linac, but were found to be unnecessary. In Project X, investigation reveals that HOM adverse effects are too small to be alarmed [2]. For the C-ADS project, on the one hand, the designed average beam current is $10 \mathrm{~mA}$, which is 10 times larger than Project $\mathrm{X}$, so that HOM effects could become much larger. On the other hand, the only operation at C-ADS is CW. The beam spectrum is well separated by $162.5 \mathrm{MHz}$, unlike in Project $\mathrm{X}$ where the beam spectrum sidebands can be as close as every 10 to $20 \mathrm{MHz}$. As a result, the HOM pass bands can be far way from the beam spectral lines, making HOM excitations relatively inefficient.

In this note, we will make simple estimate to see whether the longitudinal and transverse emittances will be enlarged by the HOM excitation. We will also make estimates of the possibility of beam breakup. Our investigation follows the examination performed by Sukhanov, et al. [3]

\section{The C-ADS}

The accelerator scheme of the C-ADS linac is illustrated in Fig. 1. It consists of the lowenergy-beam-transport (LEBT) section, the medium-energy-beam-transport (MEBT) section, the high-energy-beam-transport (HEBT) section. SRF cavities are used in both the MEBT and HEBT. Since superconducting cavities are of much higher quality factors, the corresponding shunt impedances of the HOMs are much higher and can lead to much larger collective beam dynamic effects. For this reason, we will study the HOMs of the superconducting cavities in the MEBT and HEBT only. The Ellip063 and the Ellip082 superconducting cavities are employed, respectively, in the MEBT and HEBT sections. Each of these 


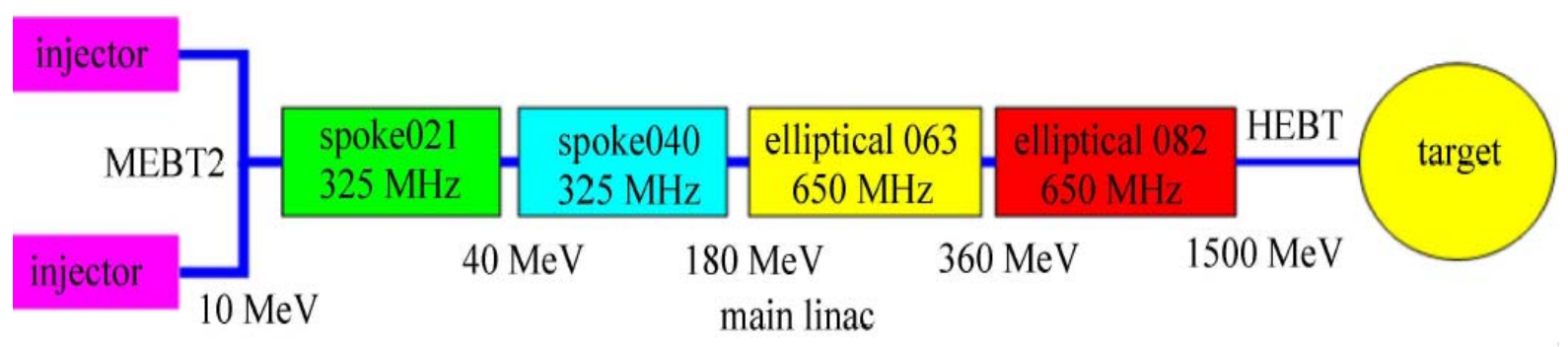

Figure 1: The layout of the C-AID linear accelerator, showing the LEBT, MEBT, and the HEBT.

cavities consists of a string of five cells. They are named Ellip063 and Ellip082 because the individual cells are of elliptical shape in the sideway projections. The former is of mean group velocity with respect to velocity of light $c$ is $\beta_{G}=0.63$, while the latter is of $\beta_{G}=0.82$. Some properties of the cavities and those of the beam are listed in Table I. The energy gain per linac period is shown in Fig. 2.

Table I: Some properties of the superconducting cavities in the MEBT and HEBT.

\begin{tabular}{lcc}
\hline \hline & Ellip063 & Ellip082 \\
\hline \hline Beam transport & MEBT & HEBT \\
No. of periods & 9 & 19 \\
No. of cavities/period $M$ & 4 & 5 \\
No. of cells/cavity & 5 & 5 \\
RF frequency $f_{\text {rf }}$ & $650 \mathrm{MHz}$ & $650 \mathrm{MHz}$ \\
Period length $L_{0}$ & $7.301 \mathrm{~m}$ & $9.62 \mathrm{~m}$ \\
Synchronous phase $\phi_{s}$ & $-12^{\circ}$ & $-10^{\circ}$ \\
Energy Range & 176 to $369.7 \mathrm{MeV}$ & 369.7 to $1501.9 \mathrm{MeV}$ \\
At entrance, $\gamma, \beta$ & $1.1875,0.5394$ & $1.3943,0.6969$ \\
At exit, $\gamma, \beta$ & $1.3943,0.6969$ & $2.5987,0.9230$ \\
Main group velocity $\beta_{G}$ & 0.63 & 0.82 \\
Main period frequency $f_{0}$ & $25.87 \mathrm{MHz}$ & $25.55 \mathrm{MHz}$ \\
Mean rf harmonic $h$ & 25.12 & 25.44 \\
Mean rf voltage per cavity $V_{\mathrm{rf}}$ & $5.5 \mathrm{MV}$ & $12.1 \mathrm{MV}$ \\
\hline \hline
\end{tabular}




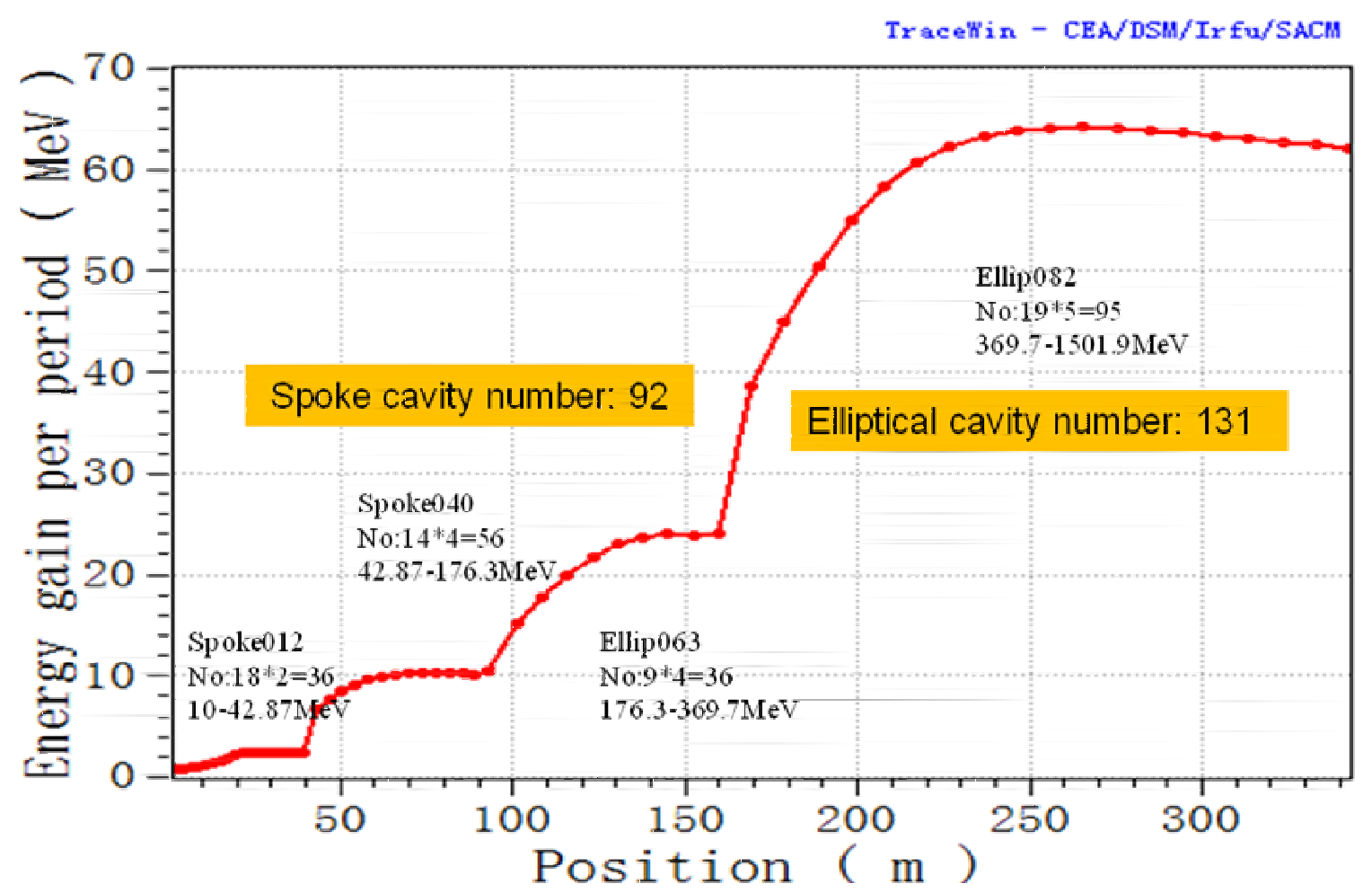

Figure 2: The energy gain per linac period obtained by simulation using the software TraceWin[6] taken from Ref. [1]. Each dot represents one period of the linac.

\section{Monopole HOM Effects}

We are going to consider the C-ADS bunches as point-bunches. This is because when entering the Ellip063 section, the C-ADS beam envelope is of $\sim 3 \mathrm{~mm}$ or $18.5 \mathrm{ps}$. The rms beam width, taking as half the envelope, is $\sigma_{t}=9.25 \mathrm{ps}$. The highest cutoff frequency is $\omega_{c} / 2 \pi \sim$ $2.77 \mathrm{GHz}$ for Ellip063 in the monopole modes. Thus the Gaussian factor $\exp \left[-\left(\omega_{c} \sigma_{t}\right)^{2} / 2\right]=$ 0.987 is just slightly different from unity. In other words, a C-ADS bunch is consider as a macroparticle of charge $e N_{b}$, where $N_{b}$ is the number of particles in the bunch. The average C-ADS beam current is $I_{b}=e N_{b} / T_{b}=10 \mathrm{~mA}$, where $T_{b}$ is the bunch separation in time and $\omega_{b} / 2 \pi$ is the beam frequency. For the C-ADS, $\omega_{b} / 2 \pi=162.5 \mathrm{MHz}$.

When a point-bunch of total charge $e N_{b}$ passes through a cavity, it excites the $n^{\text {th }}$ longitudinal HOM of frequency $\omega_{n}^{(0)}$ and internal quality factor $Q_{n}^{(0)}$. A beam particle of charge $e$ at a distance $z$ behind sees the longitudinal wake $W_{n}^{\prime(0)}(z)$ and experiences a longitudinal 
impulse $\Delta p_{n}^{(0)}(z)$, given by

$$
v \Delta p_{n}^{(0)}(z)=e^{2} N_{b} \sum_{k=-\infty}^{\infty} W_{n}^{\prime(0)}\left(z+k v T_{b}\right),
$$

where $v=\beta c$ is the nominal beam velocity. In the above, the summation implies that the beam particle sees the wakes left by not only just one bunch but all the bunches ahead. Since the wake $W_{n}^{\prime(0)}(z)$ is imbedded with the property of causality, the summation has been extended to $k=-\infty$. Because the HOMs of the superconducting cavities are of high quality factors $Q_{n}^{(0)} \gg 10^{5}$, it will be more convenient to go to the frequency space. We have

$$
v \Delta p_{n}^{(0)}(z)=e^{2} N_{b} \sum_{k=-\infty}^{\infty} \int_{-\infty}^{\infty} \frac{d \omega}{2 \pi} Z_{n}^{(0)}(\omega) e^{i \omega\left(z+k v T_{b}\right) / v},
$$

where $Z_{n}^{(0)}(\omega)$ is the corresponding longitudinal impedance of the $n^{\text {th }}$ HOM. The summation over $k$ can be performed readily to give

$$
\begin{aligned}
v \Delta p_{n}^{(0)}(z) & =e^{2} N_{b} \sum_{m=-\infty}^{\infty} \int_{-\infty}^{\infty} d \omega Z_{n}^{(0)}(\omega) e^{i \omega z / v} \delta\left(\omega T_{b}-2 \pi m\right) \\
& =e I_{b} \sum_{m=-\infty}^{\infty} Z_{n}^{(0)}\left(m \omega_{b}\right) e^{i m \omega_{b} z / v} .
\end{aligned}
$$

The longitudinal impedance of the $n^{\text {th }} \mathrm{HOM}$ can be represented by

$$
Z_{n}^{(0)}(\omega)=\frac{\left(R_{n} / Q_{n}\right)^{(0)}}{\frac{1}{Q_{n L}^{(0)}}-i\left(\frac{\omega_{n}^{(0)}}{\omega}-\frac{\omega}{\omega_{n}^{(0)}}\right)} .
$$

Here, we are using the convention which denotes time variation as $e^{i \omega t}$. When $\omega \ll \omega_{n}^{(0)}$, the impedance is of $+i$ or inductive. (This is the same as the engineering convention of $e^{j \omega t}$, with the only difference that we prefer using $i$ instead of $j$.) The $R / Q$ of a resonance in a cavity is a geometric factor of the cavity. The $\left(R_{n} / Q_{n}\right)^{(0)}$ in the above, however, includes the consideration of finite time of cavity passage. It is therefore a function of particle velocity, and is defined as

$$
\left(R_{n} / Q_{n}\right)^{(0)}(\beta)=\frac{1}{\omega_{n}^{(0)} U_{n \text { store }}^{(0)}}\left|\int_{-\infty}^{\infty} E_{n z}(r=0, z) e^{i \omega_{n}^{(0)} z / \beta c} d z\right|^{2},
$$

with $U_{n \text { store }}^{(0)}$ being the stored energy. The integration is over the electric field along the beampipe axis $(r=0)$ with transit factor taken into account. When the cavity is connected to 
the external circuit, the quality factor of a resonance decreases, and it is called the external quality factor $Q_{n L}^{(0)}$ as indicated in the denominator of Eq. (3.4). For a superconducting rf cavity in operation, $Q_{n L}^{(0)} \sim 10^{5}$, while the internal quality factor $Q_{n}^{(0)}$ is much higher. It is important to point out that as $Q_{n}^{(0)}$ decreases to $Q_{n L}^{(0)}$, the shunt impedance $R_{n}^{(0)}$ decreases to $R_{n L}^{(0)}$ as well so that $R / Q$ remains unchanged, or $R_{n L}^{(0)} / Q_{n L}^{(0)}=R_{n}^{(0)} / Q_{n}^{(0)}$.

We see in Eq. (3.3) that only frequencies at the beam multiples will contribute. Here, we are dealing with HOM frequency $\omega_{n}^{(0)} / 2 \pi \sim 1000 \mathrm{MHz}$ with external quality factor $Q_{n L}^{(0)} \sim$ $10^{5}$, or a resonance width of $\omega_{n}^{(0)} /\left(2 \pi Q_{n L}^{(0)}\right) \sim 1.6 \mathrm{kHz}$. On the other hand, the beam frequency is $\omega_{b} / 2 \pi=162.5 \mathrm{MHz}$. Thus only the beam multiple $m \omega_{b} / 2 \pi$ closest to the HOM frequency will be retained in the summation. However, one should remember that the beam multiple $-m \omega_{b} / 2 \pi$ will contribute as well. Thus a factor of two will be included if only positive frequencies are considered. If one prefers, instead of the average beam current $I_{b}$, one can substitute the beam multiple current $\tilde{I}_{b}\left(m \omega_{b}\right)=2 I_{b}(m \neq 0)$ instead. These considerations lead to longitudinal impulse imparted onto a beam particle as a result of the excitation of the $n^{\text {th }}$ longitudinal HOM,

$$
\Delta p_{n}^{(0)}=\frac{2 e I_{b}}{v} \frac{\left(R_{n} / Q_{n}\right)^{(0)}}{\frac{1}{Q_{n L}^{(0)}}+i \frac{2 \delta \omega}{\omega_{n}^{(0)}}},
$$

where $\delta \omega=m \omega_{b}-\omega_{n}^{(0)}$ and the approximation $\delta \omega / \omega_{n}^{(0)} \ll 1$ has been made. We have also substituted $z=0$ or a multiple of $v T_{b}$, because the witness beam particle considered is inside one of the bunches. The energy imparted onto the beam particle will be $U_{n}^{(0)}=v\left|\Delta p_{n}^{(0)}\right|$ If this HOM imparted impulse does not lead to an emittance increase, we must have [4]

$$
\sigma_{U_{n}} \sigma_{t} \ll \epsilon_{W t}
$$

where $\sigma_{U_{n}}=\frac{1}{\sqrt{2}} U_{n}^{(0)}$ is rms energy gain of the particle, $\sigma_{t}$ is the rms bunch length, and $\epsilon_{W t}$ is the nominal unnormalized rms longitudinal emittance [7]. We therefore require

$$
\frac{\delta \omega}{\omega_{n}^{(0)}}=\frac{e I_{b}\left(R_{n} / Q_{n}\right)^{(0)}}{U_{n}^{(0)}} \gg \frac{e I_{b}\left(R_{n} / Q_{n}\right)^{(0)} \sigma_{t}}{\sqrt{2} \epsilon_{W t}},
$$

where Eq. (3.6) has been used and $\delta \omega / \omega_{n}^{(0)} \gg 1 / 2 Q_{n}^{(0)}$ has been assumed.

Elliptical SRF Ellip063 and Ellip082 are used in C-ADS. The former is for group velocity $\beta_{G} \sim 0.63$ while the latter is for $\beta_{G} \sim 0.82$. Computation of the HOM frequencies and their corresponding $R / Q$ 's are being performed using the code CST [5], but has not been finished. The computation is very time consuming and each run can take several hours. For 
the present analysis, we are going to use the published cavity data from Project X [3], since essentially the same elliptical cavities are used in Project X. They are of the same rf frequency $f_{\mathrm{rf}}=650 \mathrm{MHz}$. Since they are used in Project X linac sections of main group velocities $0.64 c$ and $0.90 c$, they are called Ellip064 and Ellip090 instead. The dimensions of these Project X cavities are listed in Fig. 4. The monopole HOMs of Ellip064 and Ellip090 SRF cavities below

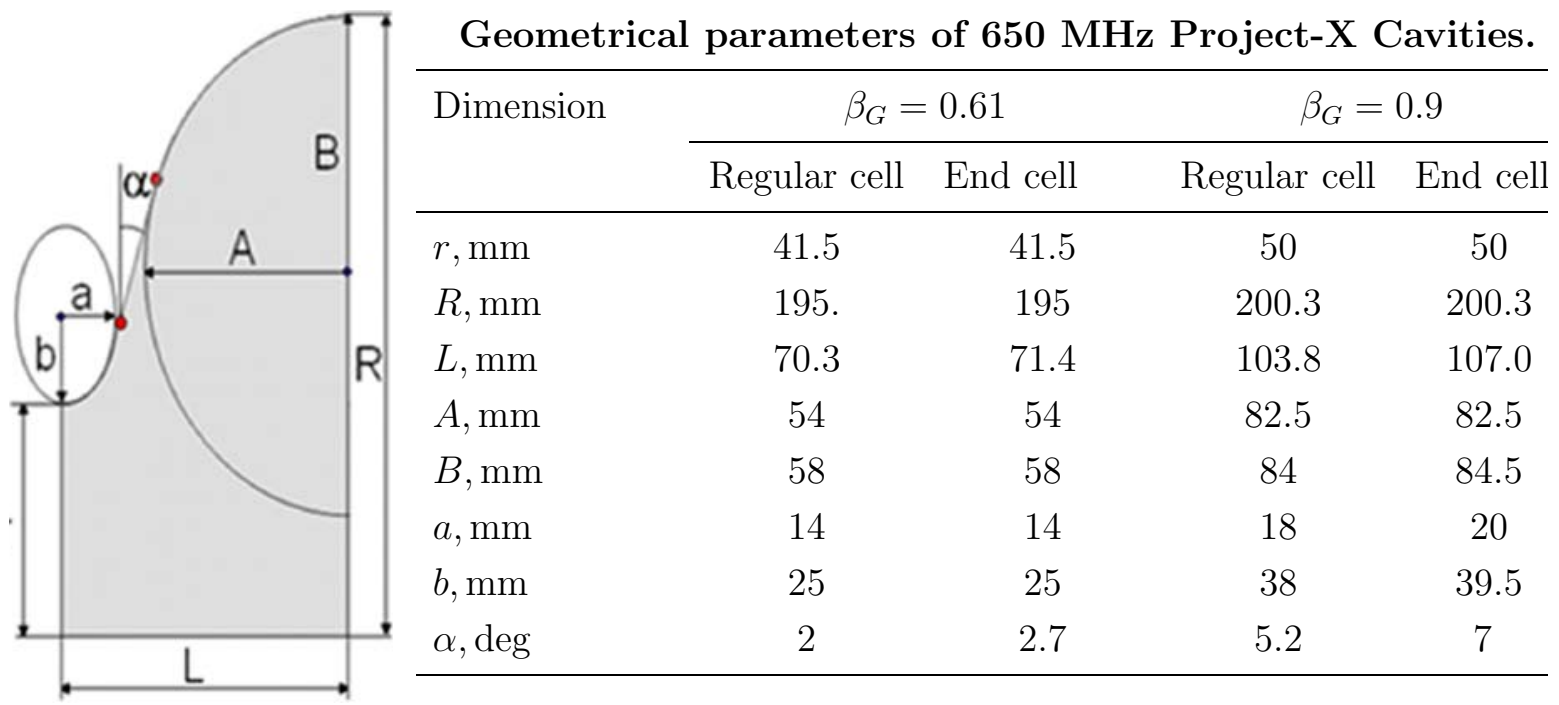

Figure 3: The dimensions of the Ellip064 and Ellip090 SRF cavities in Project X.

their respective cutoffs are plotted [3] in Fig. 4. The fundamental pass band at $\sim 650 \mathrm{MHz}$ is the acceleration band. For each pass band, there are five modes because there are five cells in each cavity. The $R / Q$ 's for monopole modes are depicted in Fig. 5. We see that there is a big variation in the values of $R / Q$ 's. The largest of them is $\left(R_{n} / Q_{n}\right)^{(0)}=130 \Omega$ for the Ellip090 HOM at frequency $\omega_{n}^{(0)} / 2 \pi=1241 \mathrm{MHz}$. The normalized rms longitudinal emittance of the C-ADS is quoted as roughly [1] $\epsilon_{z \Delta p}^{N} /\left(E_{0} / c\right)=0.23 \pi \mathrm{mm}-\mathrm{mr}$, where $E_{0}$ is the particle rest mass. At the entrance of the Ellip082 cavity section of the C-ADS main linac, $\beta=0.6969, \gamma=1 / \sqrt{1-\beta^{2}}=1.394$. In proton linacs, the unnormalized rms longitudinal emittance is often expressed as $\epsilon_{z z^{\prime}}$ with the definition $z^{\prime}=\Delta \beta / \beta$. This implies that [4] $\epsilon_{z z^{\prime}}=\epsilon_{z \Delta p}^{N} /\left(\beta \gamma^{3} E_{0}\right)=0.122 \pi \mathrm{mm}-\mathrm{mr}$, or $\epsilon_{W z}=\beta^{2} \gamma^{3} E_{0} \epsilon_{z z^{\prime}}=0.150 \pi \mathrm{keV}-\mathrm{m}$ in the energy-length unit or $\epsilon_{W t}=\beta^{2} \gamma^{3} E_{0} \epsilon_{z z^{\prime}}=0.719 \pi \mathrm{keV}$-ns in the energy-time unit. The beam envelope shows a bunch width of $2.2 \mathrm{~mm}$. Taking the half full width as $2 \sigma_{z}$, we get $\sigma_{z}=1.1 \mathrm{~mm}$ or $\sigma_{t}=5.27 \times 10^{-3} \mathrm{~ns}$. The beam spectrum consists of multiples of $\omega_{b} / 2 \pi=162.5 \mathrm{MHz}$ and the current at each multiple is $\tilde{I}_{b}\left(m \omega_{b}\right)=20 \mathrm{~mA}$. To avoid longitudinal emittance increase caused by the $\mathrm{HOM}$ at $\omega_{n}^{(0)} / 2 \pi=1241 \mathrm{MHz}$ with the largest 

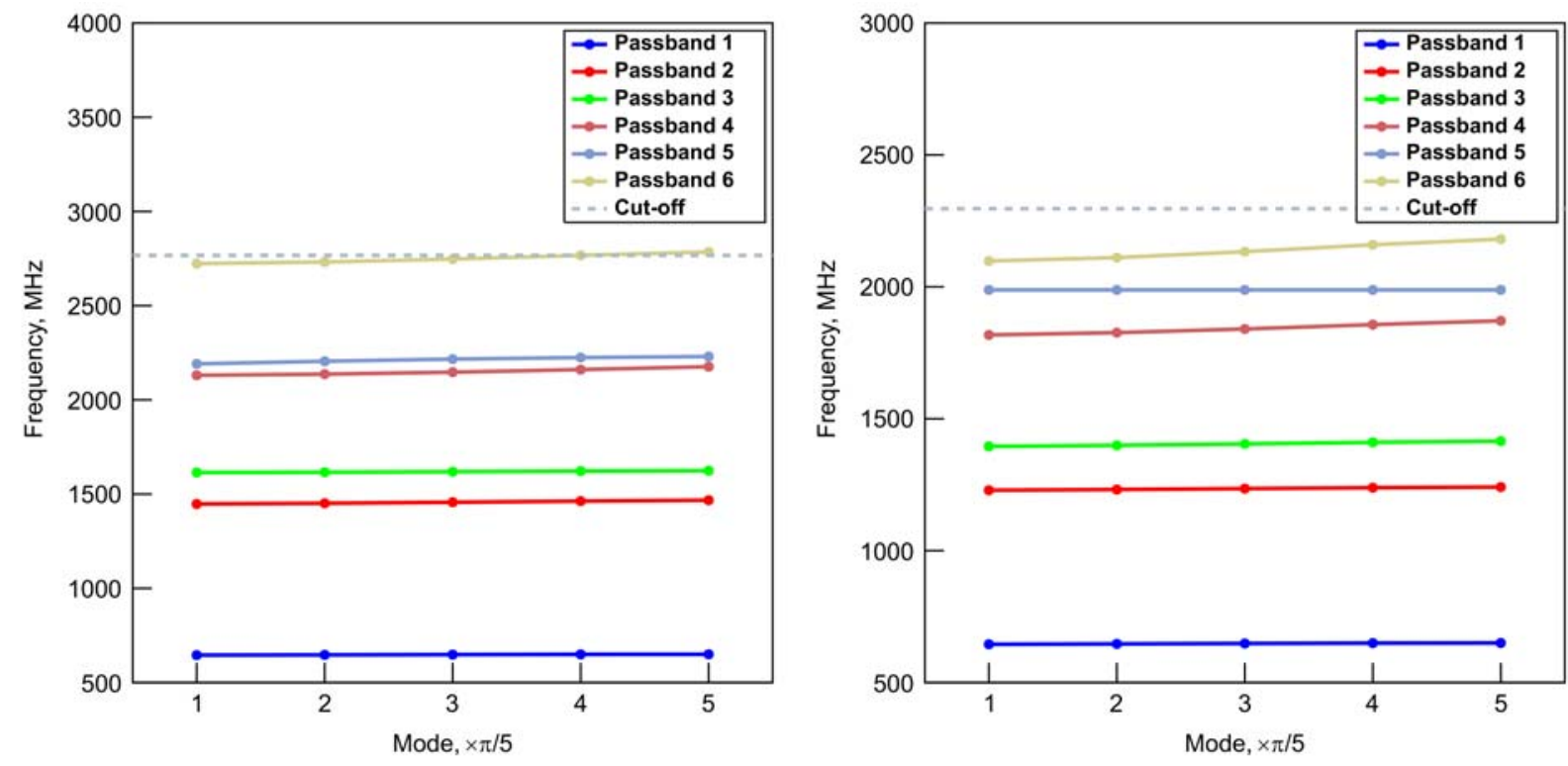

Figure 4: Monopole HOMs of the Ellip064 (left) and Ellip090 (right) SRF cavities in Project X. The five modes of each pass band are shown.

$\left(R_{n} / Q_{n}\right)^{(0)}=130 \Omega$, the HOM frequency $\omega_{n}^{(0)} / 2 \pi$ must be away from one of the beam spectral line by $\delta \omega / 2 \pi \gg 3.08 \mathrm{kHz}$, if $\tilde{I}_{b}\left(m \omega_{b}\right)=20 \mathrm{~mA}$ is substituted. Because of the mean operation velocities are slightly different in C-ADS, the actual R/Q's will usually be slightly different from those depicted in Fig. 5, unless resonances are hit. These differences, however, will be ignored in the present analysis.

The C-ADS is of CW operation and all the beam spectral lines are separated by the beam fundamental frequency $\omega_{b} / 2 \pi=162.5 \mathrm{MHz}$. The $7^{\text {th }}$ and $8^{\text {th }}$ multiples are closest to the $1241-\mathrm{MHz}$ HOM. The $7^{\text {th }}$ multiple is of frequency $1137.5 \mathrm{MHz}$ which is $103.5 \mathrm{MHz}$ away from the $1241-\mathrm{MHz} \mathrm{HOM}$, while the $8^{\text {th }}$ multiple is of frequency $1300 \mathrm{MHz}$ which is $59 \mathrm{MHz}$ away. Therefore, if the HOM at $1241 \mathrm{MHz}$ is of full spread $\Delta \omega_{n}^{(0)}=\omega_{n}^{(0)} / Q_{n L}^{(0)}$, there will not be any beam current within this spread, since the external quality factor can be of the order $Q_{n L}^{(0)} \sim 10^{5}$, and therefore this HOM will not be excited.

The above consideration is valid for one single Ellip082 cavity. There are in total 95 Ellip082 cavities in the 19 periods of the HEBT section in the C-ADS main linac. All these 95 Ellip082 cavities can never be identically manufactured. Only the accelerating mode of each cavity is carefully tuned to $650 \mathrm{MHz}$ to maximize beam acceleration. Nowadays, SRF cells and cavities have been more accurately made, and the HOM rms frequency spread can 

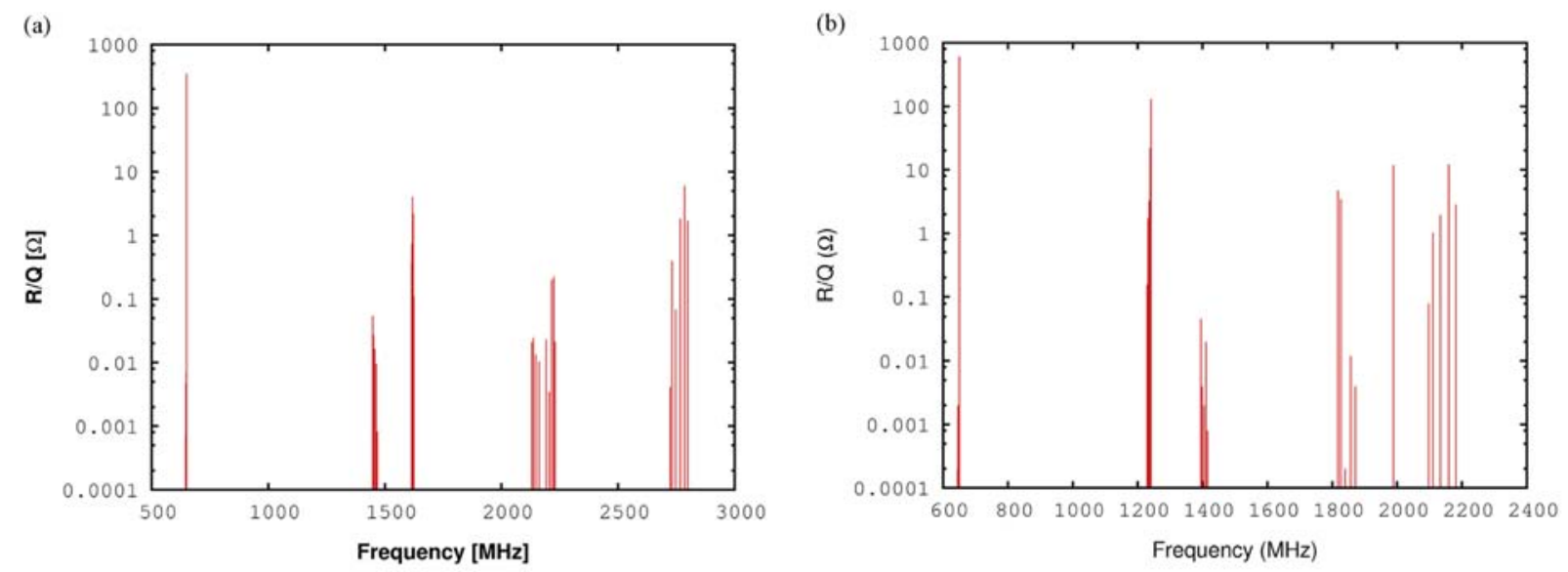

Figure 5: The monopole HOM $R / Q$ 's of the Ellip064 (left) and Ellip090 (right) SRF cavities in Project X.

be carefully controlled to less than $\sigma_{\omega_{n}} / 2 \pi \sim 5 \mathrm{MHz}$ and even as small as 1 to $2 \mathrm{MHz}$. With such a spread incorporated, the shunt impedance of the HOM $n$ mode becomes

$$
R_{\text {shunt }}^{(0)}(\omega)=\left(\frac{R_{n}}{Q_{n l}}\right)^{(0)}\left|F^{(0)}(\omega)\right|,
$$

where the form factor is

$$
F^{(0)}(\omega)=\frac{1}{\sqrt{2 \pi} \sigma_{\omega_{n}}} \int d \omega_{n}^{(0)} \frac{e^{-\left(\omega_{n}^{(0)}-\bar{\omega}_{n}^{(0)}\right)^{2} / 2 \sigma_{\omega_{n}}^{2}}}{\frac{1}{Q_{n L}^{(0)}}+i\left(\frac{\omega}{\omega_{n}^{(0)}}-\frac{\omega_{n}^{(0)}}{\omega}\right)}
$$

where $\bar{\omega}_{n}^{(0)} / 2 \pi$ is the mean frequency of HOM $n$ of all 95 Ellip082 cavities, which we may take as $1241 \mathrm{MHz}$. If we make the approximation

$$
\left(\frac{\omega}{\omega_{n}^{(0)}}-\frac{\omega_{n}^{(0)}}{\omega}\right) \approx \frac{2\left(\omega-\omega_{n}^{(0)}\right)}{\bar{\omega}_{n}^{(0)}},
$$

which is valid even when $\left(\omega-\omega_{n}^{(0)}\right) / 2 \pi$ is tens of $\mathrm{MHz}$, the form factor can be integrated in the closed form in terms of the complex error function $w($ ) (also known as Dawson function),

$$
F^{(0)}(\omega)=-\frac{\sqrt{\pi} \bar{\omega}_{n}^{(0)}}{2 \sqrt{2} \sigma_{\omega_{n}}} w\left(\frac{\omega-\bar{\omega}_{n}^{(0)}}{\sqrt{2} \sigma_{\omega_{n}}}-i \frac{\bar{\omega}_{n}^{(0)}}{2 \sqrt{2} \sigma_{\omega_{n}} Q_{n L}^{(0)}}\right)
$$

The form factor is depicted in Fig. 6 for external quality factor $Q_{n L}^{(0)}=1 \times 10^{5}$. We find that the form factor is very insensitive to the external quality factor, and the plots in Fig. 6 even 


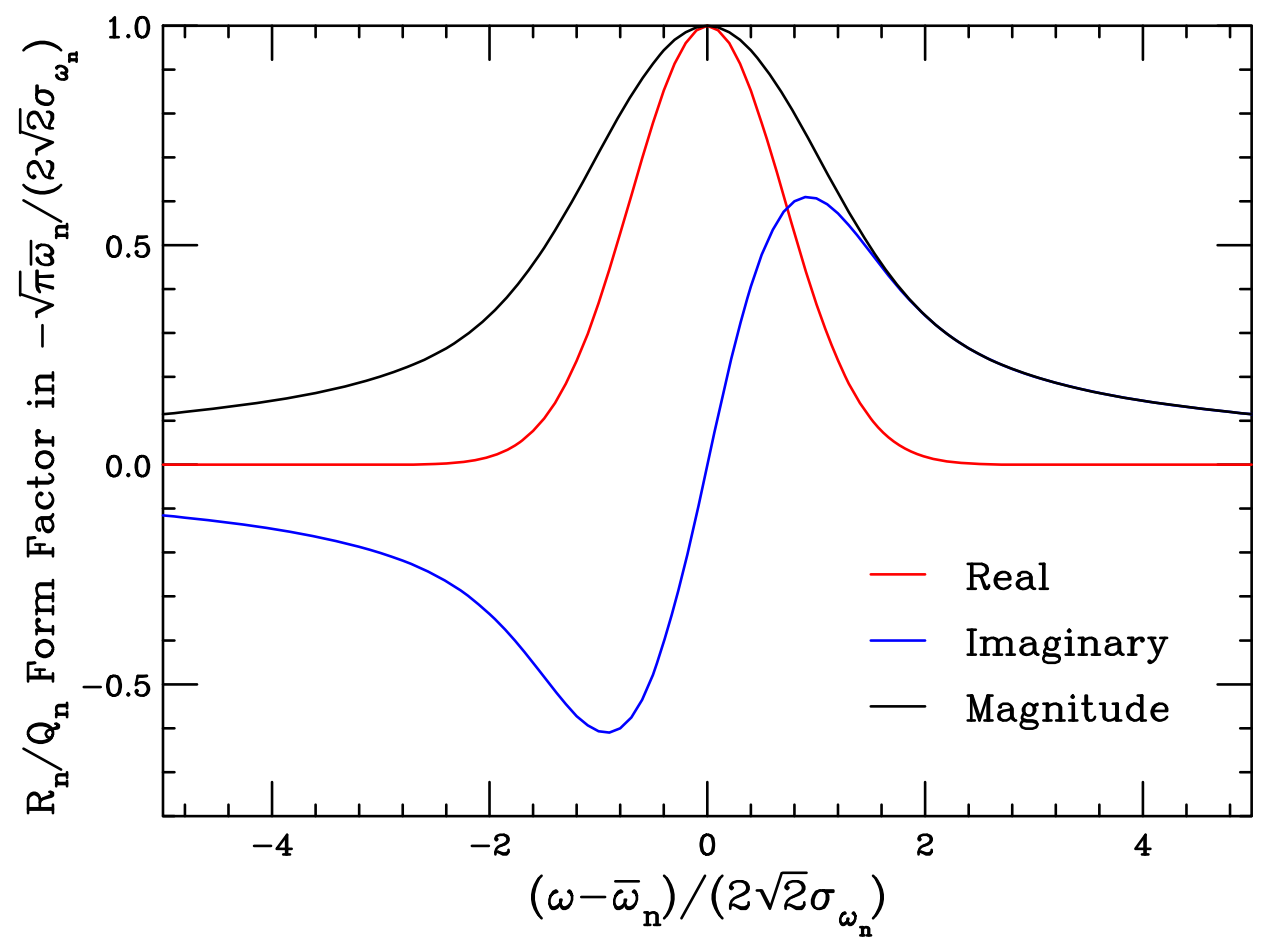

Figure 6: Plot of real and imaginary parts as well as the absolute magnitude of the $R / Q$ form factor of the SRF cavities due to imperfection of manufacturing.

do not show any visible changes as long as $Q_{n L}^{(0)} \gtrsim 1 \times 10^{3}$. This is understandable, because the HOM frequency spread due to random imperfection is very much larger than the spread due to $Q_{n L}^{(0)}$. When there is no imperfection spread, the shunt impedance right at the HOM mean frequency $\bar{\omega}_{n}^{(0)}$ is $R_{\text {shunt }}^{(0)}=R_{n}^{(0)} Q_{n L}^{(0)} / Q_{n}^{(0)}$, where $Q_{n}^{(0)}$ is the internal quality factor and is usually much higher than $Q_{n L}^{(0)}$. In the presence of the imperfection spread, however, the shunt impedance at the HOM mean frequency $\bar{\omega}_{n}^{(0)}$ is, according to Eqs. (3.9) and (3.12),

$$
R_{\text {shunt }}^{(0)}=\left(\frac{R_{n}}{Q_{n L}}\right)^{(0)} \frac{\sqrt{\pi} \bar{\omega}_{n}^{(0)}}{2 \sqrt{2} \sigma_{\omega_{n}}} .
$$

Instead of $Q_{n L}^{(0)}$, an effective external quality factor

$$
Q_{n L \mathrm{eff}}^{(0)}=\frac{\sqrt{\pi} \bar{\omega}_{n}^{(0)}}{2 \sqrt{2} \sigma_{\omega_{n}}^{(0)}}
$$

can be defined. For the monopole $\operatorname{HOM} n$ with $\omega_{n}^{(0)} / 2 \pi=1241 \mathrm{MHz}, Q_{n L \text { eff }}^{(0)}=778$, if the rms imperfection spread is $\sigma_{\omega_{n}} / 2 \pi=1 \mathrm{MHz}$. This is very much smaller than the external quality factor $Q_{n L}^{(0)}$, which can be of the order $10^{5}$. 
We have shown that the closest multiple of the bunch frequency from the mean HOM $n$ of frequency $\bar{\omega}_{n}^{(0)} / 2 \pi=1241 \mathrm{MHz}$ is the $m=8^{\text {th }}$ multiple and is $59 \mathrm{MHz}$ away. The $R / Q$ form factor at the $8^{\text {th }}$ multiple is $\left|F^{(0)}\left(8 \omega_{b}\right)\right|=10.5$. Recall that the HOM $n$ of an individual SRF cavity can have a very large quality factor $Q_{n}$. Because of the coupling with external impedances, the quality factor is largely reduced to the external quality factor $Q_{n L}^{(0)}$, which may be of the order of $10^{5}$. When many superconducting cells are considered, because of the imperfection in manufacturing, there is a spread in the HOM frequency, for example $\sigma_{\omega_{n}} / 2 \pi \sim 1 \mathrm{MHz}$. This spread effectively lowers $Q_{n L}^{(0)}$ to an effective value of $Q_{n L \text { eff }}^{(0)} \sim 778$. Because the closest bunch multiple spectral line is $59 \mathrm{MHz}$ away from the mean HOM frequency, the coupling of this $8^{\text {th }}$ bunch current with the HOM is further reduced through the form factor of the shunt impedance from $Q_{n L \text { eff }}^{(0)} \sim 778$ to $\left|\mathcal{R} e F^{(0)}\left(8 \omega_{b}\right)\right|=10.5$. The average voltage induced per cavity at the Ellip082 SRF in the HOM of frequency $1241 \mathrm{MHz}$ is therefore

$$
V_{n}^{(0)}\left(8 \omega_{b}\right)=\frac{U_{n}^{(0)}\left(8 \omega_{b}\right)}{e}=\tilde{I}_{b}\left(8 \omega_{b}\right)\left(\frac{R_{n}}{Q_{n}}\right)^{(0)}\left|\mathcal{R} e F^{(0)}\left(8 \omega_{b}\right)\right|=27.4 \mathrm{~V} .
$$

The average power loss per cavity for this monopole HOM is

$$
P_{\mathrm{loss}}^{(0)}=\frac{V_{n}^{(0)^{2}}}{\left(\frac{R_{n}}{Q_{n}}\right)^{(0)}\left|\mathcal{R e} F^{(0)}\left(8 \omega_{b}\right)\right|}=0.55 \mathrm{~W} .
$$

\section{Frequency Spread of Beam Frequency}

There is a spread in the velocity of the particles in a bunch, which will lead to a spread in the frequency of the beam. In the previous section, we found that the unnormalized rms longitudinal emittance at the entrance of the Ellip082 section is $\epsilon_{z z^{\prime}}=0.122 \pi \mathrm{mm}-\mathrm{mr}$, and the rms bunch length is $\sigma_{z}=1.1 \mathrm{~mm}$. Thus the rms spread in particle velocity is $\sigma_{\Delta \beta / \beta}=\epsilon_{z z^{\prime}} / \sigma_{z}=0.11 \mathrm{mr}$. The beam frequency is $\omega_{b} / 2 \pi=\beta c / L_{\mathrm{sep}}$, where $L_{\mathrm{sep}}$ is the nominal distance between successive bunches. The spread in beam frequency is therefore

$$
\Delta \omega_{b}=-\frac{\beta c}{L_{\text {sep }}} \frac{\Delta \beta}{\beta}=-\omega_{b} \frac{\Delta \beta}{\beta} .
$$

Thus the rms spread in beam frequency is $\sigma_{\omega_{b}} / 2 \pi=0.018 \mathrm{MHz}$, and $\sigma_{m \omega_{b}} / 2 \pi=0.018 \mathrm{~m} \mathrm{MHz}$ for the $m^{\text {th }}$ multiple. Since the $1241-\mathrm{MHz}$ is closest to the $8^{\text {th }}$ multiple, the rms spread of the current component $\tilde{I}_{b}\left(8 \omega_{b}\right)$ is $0.14 \mathrm{MHz}$. Thus the HOM is far from a multiple beam 
current frequency only when the separation is very much larger than $\left(\sigma_{\omega_{n}}+\sigma_{m \omega_{b}}\right) / 2 \pi$, which is about $1.14 \mathrm{MHz}$ in the present case, if we assume $\sigma_{\omega_{n}} / 2 \pi=1 \mathrm{MHz}$. The derivation in the previous section will not be affected, because the separation of the HOM from the $8^{\text {th }}$ multiple of the beam frequency is $59 \mathrm{MHz}$, which is very much bigger.

\section{Dipole HOM Effects}

Besides monopole, dipole HOMs can also be excited in SRF cavities. If the dipole-mode energy imparted to the beam particles is large enough, transverse emittances can increase and eventually lead to beam breakup. Here, our analysis follows closely that for the monopole HOMs.

Let us concentrate on the horizontal phase space. Consider a point-bunch of total charge $e N_{b}$ passes through a cavity at a horizontal offset $x_{1}$. It excites a dipole HOM $n$ of the cavity of frequency $\omega_{n}^{(1)} / 2 \pi$ and internal quality factor $Q_{n}^{(1)}$. A beam particle of charge $e$ at a distance behind sees the horizontal wake $W_{n}^{(1)}(z)$ and experiences a horizontal impulse $\Delta p_{n}^{(1)}(z)$, given by

$$
v \Delta p_{n}^{(1)}(z)=e^{2} N_{b} x_{1} \sum_{k=-\infty}^{\infty} W_{n}^{(1)}\left(z+k v T_{b}\right)
$$

Introducing the transverse impedance

$$
Z_{n}^{(1)}(\omega)=\frac{c}{\omega_{n}^{(1)}} \frac{\left(R_{n} / Q_{n}\right)^{(1)}}{\frac{1}{Q_{n L}^{(1)}}-i\left(\frac{\omega_{n}^{(1)}}{\omega}-\frac{\omega}{\omega_{n}^{(1)}}\right)}
$$

summing over $k$ and retaining only the beam multiple $m \omega_{b}$ closest to the HOM frequency under consideration, we obtain

$$
\Delta p_{n}^{(1)}=\frac{2 i e I_{b}}{c} x_{1} Z_{n}^{(1)}\left(m \omega_{b}\right)=\frac{2 i e I_{b} x_{1}}{\omega_{n}^{(1)}} \frac{\left(R_{n} / Q_{n}\right)^{(1)}}{\frac{1}{Q_{n L}^{(1)}}+i \frac{2 \delta \omega}{\omega_{n}^{(1)}}}
$$

where $\delta \omega=m \omega_{b}-\omega_{n}^{(1)}$, only positive frequencies are considered, and the assumption $\delta \omega / \omega_{n}^{(1)} \ll 1$ has been made. In the above, the dipole HOM shunt impedance over quality factor is a function of beam velocity $\beta$ and is defined as

$$
\left(\frac{R_{n}}{Q_{n}}\right)^{(1)}(\beta)=\frac{1}{\omega_{n}^{(1)} U_{n \text { store }}^{(1)}}\left|\frac{1}{x_{1}} \int_{-\infty}^{\infty} E_{n z}\left(r=x_{1}, z\right) e^{i \omega_{n}^{(1)} z / \beta c} d z\right|^{2},
$$


with $U_{n s t o r e}^{(1)}$ being the energy stored per cycle. The external quality factor is $Q_{n L}^{(1)}$.

This horizontal impulse imparted on the beam particle lead to an increase of the defection of the beam in the horizontal space space by

$$
\Delta x^{\prime}=\frac{\left|\Delta p_{n}^{(1)}\right|}{p}=\frac{2 e I_{b} x_{1}}{p \omega_{n}^{(1)}}\left|\frac{\left(R_{n} / Q_{n}\right)^{(1)}}{\frac{1}{Q_{n L}^{(1)}}-i \frac{2 \delta \omega}{\omega_{n}^{(1)}}}\right|,
$$

where $p$ is the nominal longitudinal momentum of the beam. In order that this transverse kick will not affect the horizontal emittance of the bunch, we require

$$
\Delta \sigma_{x^{\prime}} \ll \frac{\epsilon_{x}}{\sigma_{x}}=\frac{\epsilon_{x}}{\sqrt{\epsilon_{x} \beta_{x}}}=\sqrt{\frac{\epsilon_{x}}{\beta_{x}}},
$$

where $\Delta \sigma_{x^{\prime}}=\Delta x^{\prime} / \sqrt{2}$ and $\epsilon_{x}$ is the unnormalized rms horizontal emittance of the bunch, while $\sigma_{x}$ is the rms horizontal beam width and $\beta_{x}$ is horizontal betatron function at the cavity. With the help of Eq. (5.22), this requirement translates into

$$
\delta \omega=\frac{e I_{b} c x_{1}\left(R_{n} / Q_{n}\right)^{(1)}}{p \Delta x^{\prime}} \gg \frac{e I_{b} c x_{1}\left(R_{n} / Q_{n}\right)^{(1)}}{\sqrt{2} \gamma \beta E_{0} \sqrt{\epsilon_{x} / \beta_{x}}}
$$

where $E_{0}$ is the rest energy of the beam particle. The assumption that in most cases $\delta \omega / \omega_{n}^{(1)}=\left(\omega-\omega_{n}^{(1)}\right) / \omega_{n}^{(1)} \gg 1 / 2 Q_{n L}^{(1)}$ has been used.

The dipole eigenmodes of the SRF cavity are shown on the left for Ellip064 and on the right for Ellip090 in Fig. 7. The corresponding dipole $R / Q$ 's are shown in Fig. 8. For each pass band, there are five modes because there are five cells in each cavity. The $R / Q$ 's for the dipole modes are depicted in Fig. 8.

The most offensive dipole HOM is that of frequency $\omega_{n}^{(1)}=1376 \mathrm{MHz}$ with $\left(R_{n} / Q_{n}\right)^{(1)}=$ $60 \mathrm{k} \Omega / \mathrm{m}^{2}$ in the Ellip090 cavity. At the entrance of the Ellip082 section in the C-ADS main linac, the normalized horizontal $\mathrm{rms}$ emittance is $\epsilon_{x}^{N}=0.26 \pi \mathrm{mm}-\mathrm{mr}$. The beam energy corresponds to $\gamma=1.3943$ and $\beta=0.6969$. The unnormalized horizontal rms emittance is therefore $\epsilon_{x}=0.269 \pi \mathrm{mm}-\mathrm{mr}$. The beam envelope has the horizontal size of $5 \mathrm{~mm}$. The rms horizontal beam size, taken as half of the envelope size, is $\sigma_{x}=2.5 \mathrm{~mm}$, which corresponds to the betatron function $\beta_{x}=23.4 \mathrm{~m}$. To avoid emittance increase, the dipole HOM frequency must be away from a beam spectral line by $\delta \omega / 2 \pi=207 \mathrm{~Hz}$, if the multiple beam current of $\tilde{I}_{b}\left(m \omega_{b}\right)=2 I_{b}=20 \mathrm{~mA}$ is substituted and the beam passes through the cavity at an offset of $x_{1}=1 \mathrm{~mm}$. This dipole HOM frequency is in between the $8^{\text {th }}$ and the $9^{\text {th }}$ multiples of 

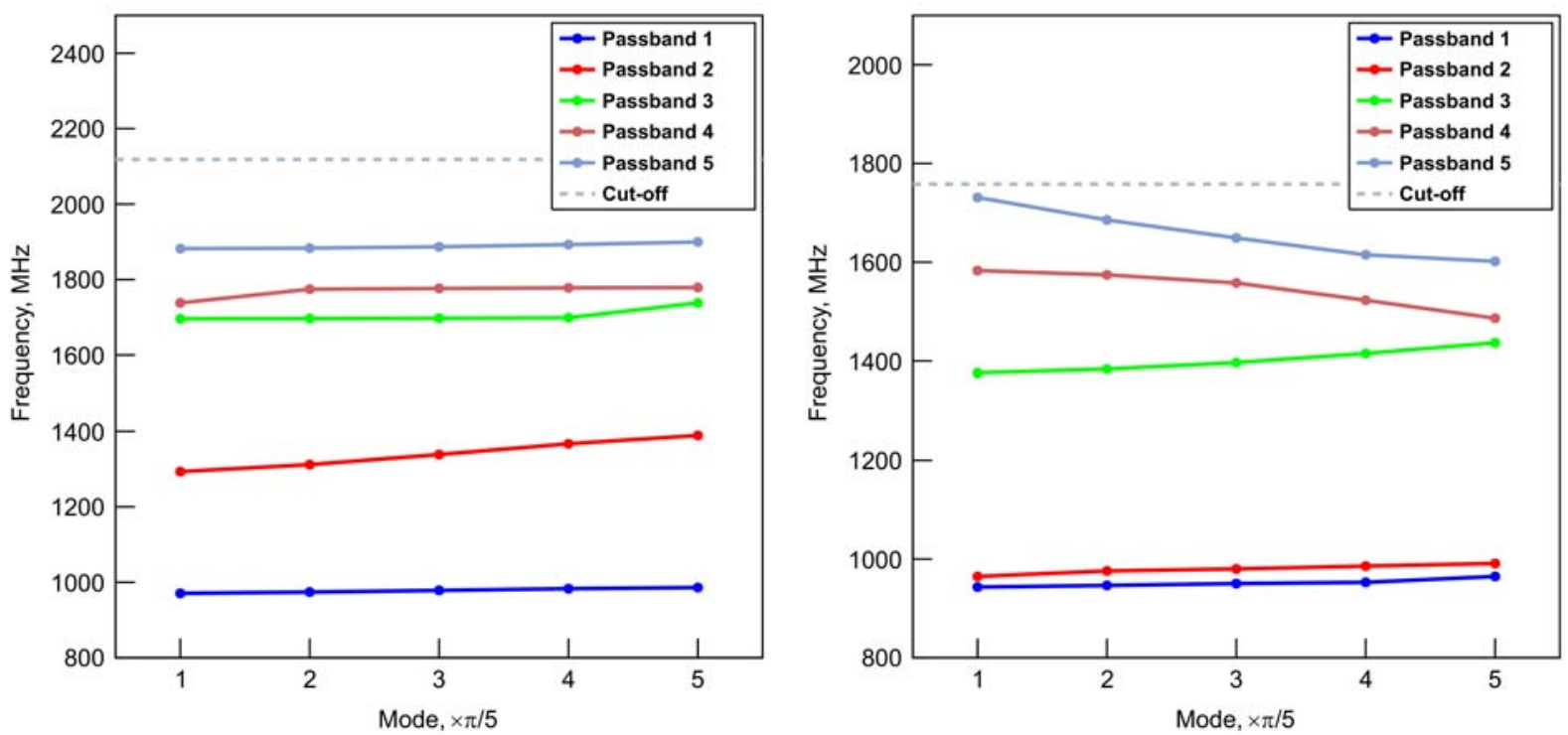

Figure 7: Dipole HOMs of the Ellip064 (left) and Ellip090 (right) SRF cavities in Project X. The five modes of each pass band are shown.

the bunch frequency. The $8^{\text {th }}$ multiple is $76 \mathrm{MHz}$ away from the dipole $\mathrm{HOM}$ while the $9^{\text {th }}$ is $86.5 \mathrm{MHz}$ away.

If the frequency spread due to manufacturing of all the 95 Ellip082 cavities are taken into account, we are seeing the same form factor in Eq. (3.12), but with the superscript (0) replaced by (1). Thus the dipole shunt impedance is

$$
R_{\text {shunt }}^{(1)}(\omega)=\left(\frac{R_{n}}{Q_{n}}\right)^{(1)}\left|F^{(1)}(\omega)\right| .
$$

Right at the mean dipole HOM frequency $\bar{\omega}_{n}$, the effective quality factor is $Q_{n L \text { eff }}^{(1)}=$ $\left|\mathcal{R e} F^{(1)}\left(\bar{\omega}_{n}\right)\right|=862$ when $\sigma_{\omega_{n}} / 2 \pi=1 \mathrm{MHz}$ is assumed. At the $8^{\text {th }}$ multiple of the beam frequency the form factor is $\left|\mathcal{R} e F^{(1)}\left(8 \omega_{b}\right)\right|=9.05$. If the dipole HOM frequency is right on a multiple bunch frequency, the energy imparted to a beam particle is

$$
U_{n}^{(1)}=\frac{x_{1} e \tilde{I}_{b}\left(8 \omega_{b}\right) c}{\omega_{n}^{(1)}}\left(\frac{R_{n}}{Q_{n}}\right)^{(1)} Q_{n L \text { eff }}^{(1)}=23.5 \mathrm{eV} .
$$

Since the $8 \omega_{b}$ component is actually $76 \mathrm{MHz}$ away, the energy imparted to a beam particle is instead

$$
U_{n}^{(1)}=\frac{x_{1} e \tilde{I}_{b}\left(8 \omega_{b}\right) c}{\omega_{n}}\left(\frac{R_{n}}{Q_{n}}\right)^{(1)}\left|\mathcal{R} e F^{(1)}\left(8 \omega_{b}\right)\right|=0.377 \mathrm{eV}
$$


(a)

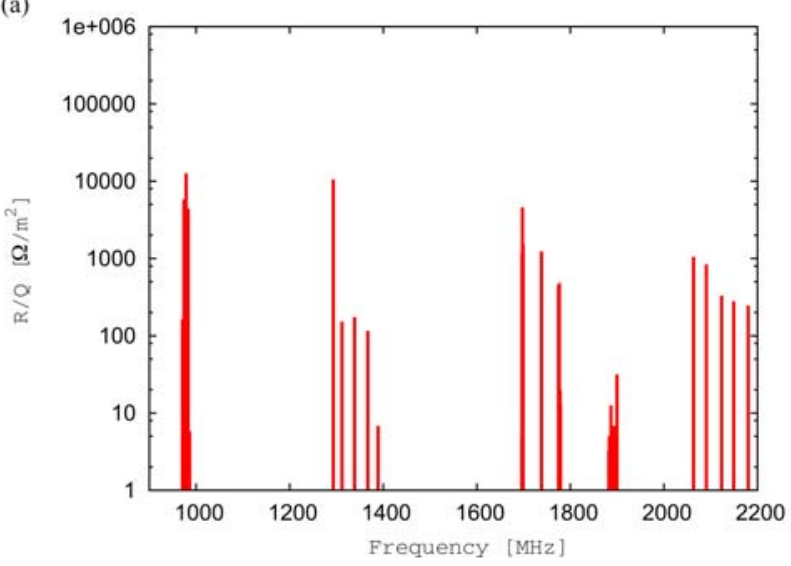

(b)

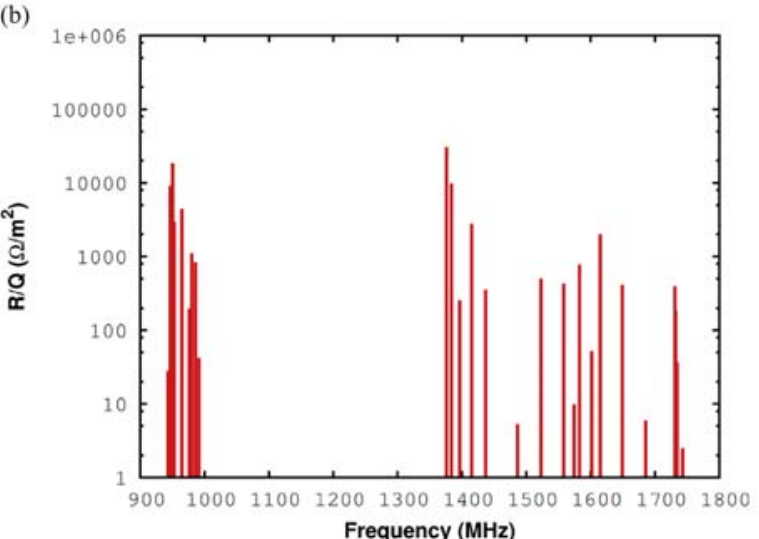

Figure 8: The $R / Q$ 's HOMs of the Ellip064 (left) and Ellip090 (right) SRF cavities in Project X.

The power dissipated can be obtained by multiplying the above voltage by $\tilde{I}_{b}\left(8 \omega_{b}\right)$. This gives $P_{\text {loss }}^{(1)}=0.0075 \mathrm{~W}$ per cavity. However, if the dipole HOM frequency is right on top of a multiple bunch frequency, this loss would become $0.047 \mathrm{~W}$ per cavity.

\section{Collective Effects}

Let us go to the time domain and sum up the HOM voltage induced by an infinite train of bunches. For the dipole HOM, dipole wake is left by an infinite train of bunches traversing the cavity at an offset $x_{1}$. A beam particle of charge $e$ passing the cavity at whatever offset will experience a dipole transverse impulse

$$
\begin{aligned}
\Delta p_{n}^{(1)} & =e^{2} N_{b} x_{1}\left(\frac{R_{n}}{Q_{n}}\right)^{(1)} \sum_{k=0}^{\infty} e^{-k \omega_{n}^{(1)} T_{b} / 2 Q_{n L}^{(1)}} \sin k \omega_{n}^{(1)} T_{b} \\
& =e^{2} N_{b} x_{1} \operatorname{Im} \frac{\left(R_{n} / Q_{n}\right)^{(1)}}{1-\exp \left(-\omega_{n}^{(1)} T_{b} / 2 Q_{n L}^{(1)}+i \omega_{n}^{(1)} T_{b}\right)},
\end{aligned}
$$

where $\omega_{b} / 2 \pi=T_{b}^{-1}$ is the beam frequency. The summation covering only nonnegative $k$ is the result of causality. If the HOM is a multiple of the beam frequency, the denominator will vanish when the HOM quality factor $Q_{n L}^{(1)} \rightarrow \infty$. Physically, the induced voltage adds up because the HOM is in phase with the beam. However, if the HOM is far from a multiple of the beam frequency, the accumulated induced voltage will be much smaller. This is a reflection of the same far-away-from-multiple effect we saw in the frequency domain. 
Some comments are necessary. Since this is a linac, every bunch performs the same betatron oscillation with the same initial phase, except for some small variation because of the small spreads of original position, energy, etc. As is shown in Fig. 9, every bunch passes through one particular cavity at roughly the same offset $x_{1}$. It is because of this that

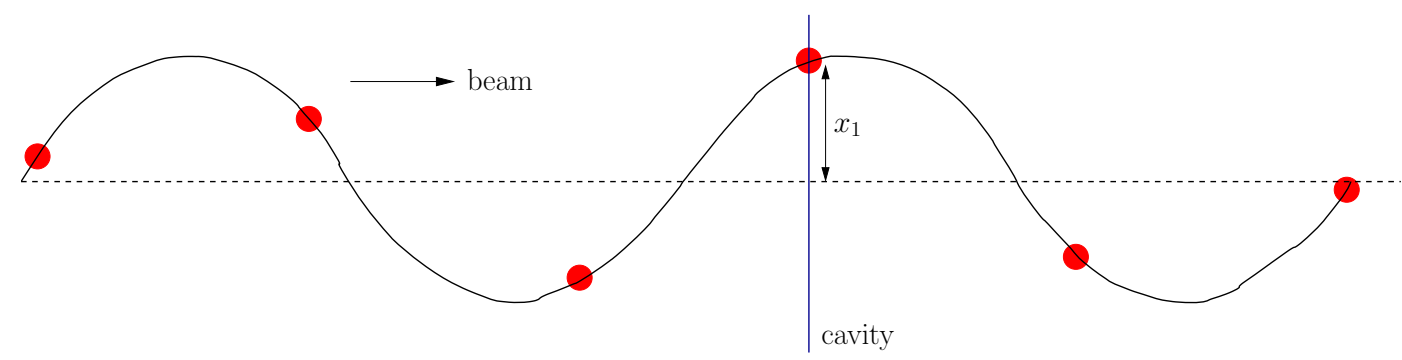

Figure 9: Every bunch passes through the cavity at the same offset $x_{1}$, except for the small variation due to the small spreads of original position, energy, etc.

the bunch offset $x_{1}$ is not included within the summation sign in Eq. (6.28). However, $x_{1}$ is a function of cavity position, implying that the bunches pass through another cavity at another offset.

The excitation of the $n^{\text {th }}$ transverse HOM of $M$ SRF cavities in a linac period of length $L_{0}$ by former bunch passage initiates a transverse impulse $M \Delta p_{n}^{(1)}$ or an average transverse force $M \Delta p_{n}^{(1)} / L_{0}$ on the test particle. Since this average transverse force is linear with the offset $x_{1}$ of beam passage, we can define an equivalent transverse force quadrupole gradient

$$
K=\frac{v \Delta p_{n}^{(1)} M}{\gamma \beta^{2} E_{0} L_{0}}=\frac{e^{2} N_{b} M c}{\gamma \beta E_{0} L_{0}} \operatorname{Im} \frac{\left(R_{n} / Q_{n}\right)^{(1)}}{1-\exp \left(-\omega_{n}^{(1)} T_{b} / 2 Q_{n L}+i \omega_{n}^{(1)} T_{b}\right)},
$$

where $E_{0}$ is the particle rest energy.

Because the operation is CW, the bunch train is infinite. If the dipole beam loading effects are not too severe, a steady state will be reached. The equation of motion governing the steady-state transverse motion of a bunch can therefore be written as

$$
\frac{1}{\gamma \beta} \frac{d}{d s}\left(\gamma \beta \frac{d x}{d s}\right)+k_{\beta}^{2} x=K x
$$

where $k_{\beta}=1 / \beta_{x}$ is the betatron wave number of a period of the main-linac lattice, and $\beta_{x}$ is the average horizontal betatron function of the linac lattice. The quadrupoles in the lattice give rise to the transverse focusing force $k_{\beta}^{2} x$ responsible for betatron oscillation. The dipole 
HOM impulse-induced $K x$, on the other hand, is repulsive. In order that stable betatron motion is ensured, we must require $k_{\beta}^{2}>K$. Otherwise, the bunches will be deflected more and more transversely while traveling down the linac. This phenomenon is called beam breakup. A beam breakup parameter $\Upsilon^{(1)}$ can be defined:

$$
\Upsilon^{(1)}=\frac{K}{k_{\beta}^{2}}=\frac{e^{2} N_{b} M c}{k_{\beta}^{2} \gamma \beta E_{0} L_{0}} \frac{e^{-\theta} \sin \phi\left(R_{n} / Q_{n}\right)^{(1)}}{1-2 e^{-\theta} \cos \phi+e^{-2 \theta}} .
$$

Here we have made the abbreviation $\theta=\omega_{n}^{(1)} T_{b} / 2 Q_{n L}^{(1)}$, representing the decay index and should be a small number, whereas $\phi=\omega_{n}^{(1)} T_{b}$ represents the dipole HOM phase advance in one beam period. Betatron motion will not be perturbed by much if $\Upsilon^{(1)} \ll 1$. When $\Upsilon^{(1)} \gtrsim 1$, betatron focusing will be lost and beam breakup occurs. Since $\Upsilon^{(1)}$ is inversely proportional to $\gamma \beta^{2}$, beam breakup will occur most easily at low beam momentum. In our analysis below, we will be looking at the entrance of the Ellip082 section, where $\gamma=1.3943$ and $\beta=0.6969$.

If the HOM frequency $\omega_{n}^{(1)} / 2 \pi$ is closest to the $m^{\text {th }}$ multiple of the beam frequency, let $\omega_{n}^{(1)}=m \omega_{b}+2 \pi \Delta$ and denote $\phi=\omega_{n}^{(1)} T_{b}=\left(m \omega_{b}+2 \pi \Delta\right) T_{b}=2 \pi\left(m+\Delta T_{b}\right)$, where $\Delta=\left(\omega_{n}^{(1)}-m \omega_{b}\right) / 2 \pi$ is the separation of the dipole HOM frequency under consideration from the closest beam frequency multipole.

When $\Delta=0$ or when the HOM frequency is an exact multiple of the beam frequency, the kick in the transverse direction generated by successive bunch passages just adds up in phase and will appear to diverge if the quality factor $Q_{n L}^{(1)} \rightarrow \infty$. However, the beam breakup parameter $\Upsilon^{(1)}$ is exactly zero. This is understandable because the dipole wake starts off from $\sin \omega_{n}^{(1)} z / v$. But this does not mean that there will not be any transverse kicks imparted to the beam particles passing by. Actually, $\Upsilon^{(1)}$ assumes a maximum when $\omega_{n}^{(1)}$ is just above $m \omega_{b}$ and a minimum when $\omega_{n}$ just below $m \omega_{b}$. The extrema occur when $\cos \phi=\operatorname{sech} \theta$. When $Q_{n L}^{(1)} \gg 1$, which is usually the case, the maximum and minimum occur at $\Delta= \pm \omega_{n}^{(1)} /\left(4 \pi Q_{n L}^{(1)}\right)$, which is $\pm 6.50 \mathrm{kHz}$ in this situation. Correspondingly, the maximum and minimum dipole-wake beam breakup parameter are $\Upsilon^{(1)} \sim 0.7$, rather close to the threshold of beam breakup. In the computation we have used an average betatron function of $\beta_{x}=23.4 \mathrm{~m}$ or the betatron wave number is $k_{\beta}=1 / \beta_{x}=0.0427 \mathrm{~m}^{-1}$. The dipole HOM that has the largest $\left(R_{n} / Q_{n}\right)^{(1)}=60 \mathrm{k} \Omega / \mathrm{m}^{2}$ in the Ellip090 cavity at $\omega_{n}^{(1)} / 2 \pi=1376 \mathrm{MHz}$ is assumed. A stronger betatron focusing will definitely help to counteract the transverse dipole wake accumulation. Fortunately, there is no alarm, because Fig. 10 shows that the maximum and minimum peaks occur only when the HOM frequency differs from a multiple 


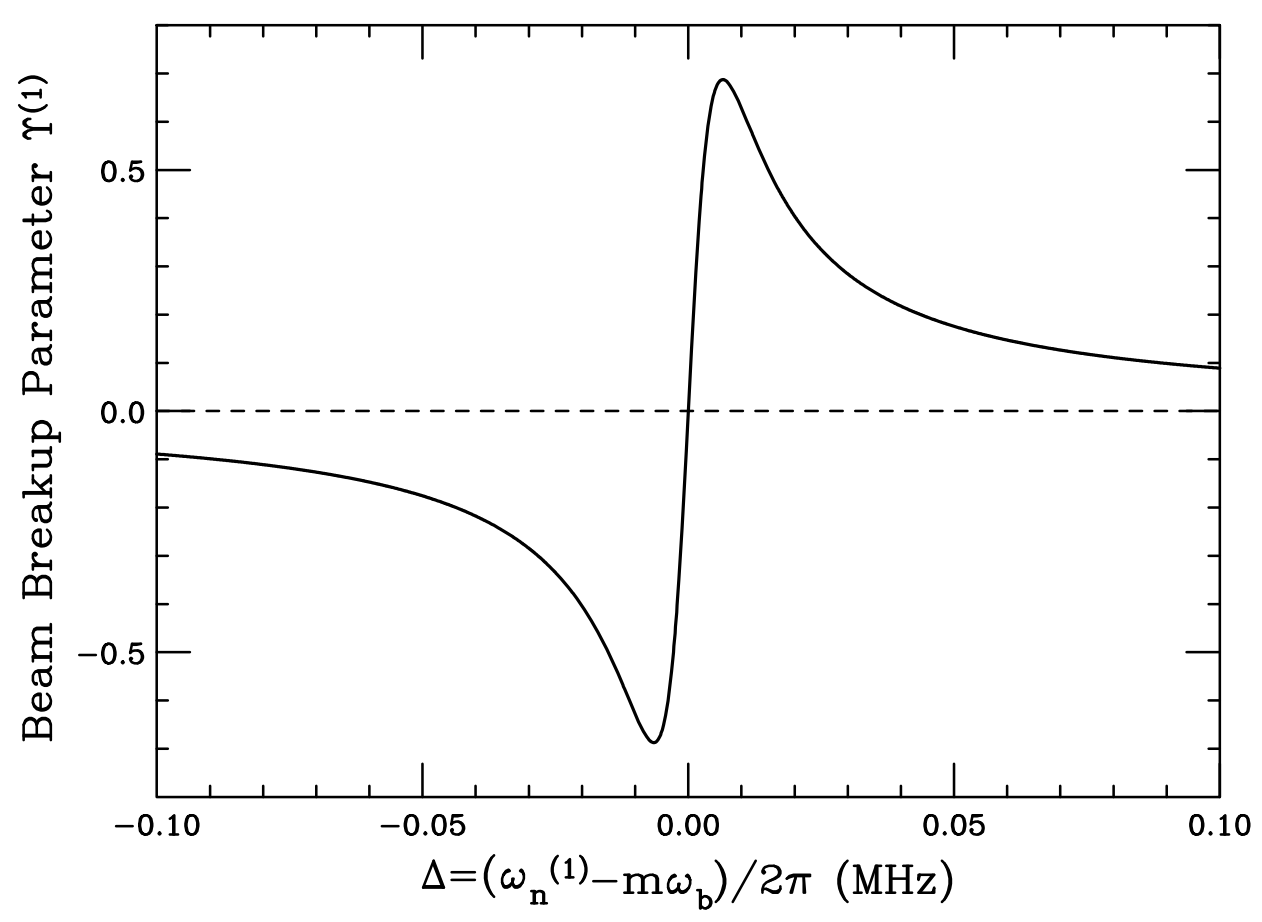

Figure 10: Plot of transverse beam offset growth ratio $\Upsilon^{(1)}$ for the Ellip082 section of the main linac, caused by cumulative transverse kick voltages excited by CW bunches of frequency $\omega_{b} / 2 \pi=$ 162.5 $\mathrm{MHz}$ versus the deviation of the $\mathrm{HOM}$ frequency from the nearest multiple of the beam frequency. The external quality factor of $Q_{n L}^{(1)}=1 \times 10^{5}$ has been assumed.

of the beam frequency by $\pm 6.5 \mathrm{kHz}$. Away from this region, $\Upsilon^{(1)}$ rolls off rapidly and is less than 0.01 when $\Delta>0.1 \mathrm{MHz}$. In our situation, the most offensive dipole HOM is $\Delta=76 \mathrm{MHz}$ away from the closest $8^{\text {th }}$ multiple of the beam frequency. The beam breakup parameter from accumulated dipole wake is $\Upsilon^{(1)}=1.8 \times 10^{-5}$ only at the entrance of the Ellip082 section.

\section{Monopole HOM Effects on Longitudinal Motion}

Beam particles execute synchrotron oscillations for longitudinal focusing. The extra HOM induced voltage experienced by the beam particles may shift the position of the beam center, and can be viewed as effectively shifting the synchrotron phase.

The first longitudinal equation of motion is for the particle arrival time $\tau$ at some 
designated point in a period of the main linac,

$$
\frac{d \tau}{d s}=-\frac{\eta \Delta E}{v_{0} \beta^{2} \gamma E_{0}}=\frac{\Delta E}{c \beta^{3} \gamma^{3} E_{0}}
$$

where $\Delta E$ is the energy offset of the beam particle and $\eta=-\gamma^{-2}$ is the slip factor in the absence of dispersion. The energy of the beam particle increases because of the acceleration field in the SRF cavities plus the impulse $\Delta p_{n}^{(0)}$ coming from the wake left by the excitation of the $n^{\text {th }}$ monopole HOM in the cavities. The energy change equation of motion is

$$
\frac{d \Delta E}{d s}=\frac{e M V_{\mathrm{rf}}}{L_{0}}\left[\cos \left(\phi_{s}-\omega_{\mathrm{rf}} \tau\right)-\cos \phi_{s}\right]+\frac{e M v \Delta p_{n}^{(0)}}{L_{0}},
$$

where $M$ is the number of SRF cavities in a period, $V_{\mathrm{rf}}$ is the rf voltage per cavity and $\omega_{\text {rf }} / 2 \pi$ is the rf frequency, while $\phi_{s}$ is the synchrotron phase measured from the peak of the sinusoidal rf wave. The last term represents the energy from the excited HOM in the $M$ cavities of the period. The two equations combine to give

$$
\frac{d^{2} \tau}{d s^{2}}+\frac{4 \pi^{2} \nu_{s}^{2}}{L_{0}^{2}}\left(\tau-\frac{v \Delta p_{n}^{(0)} L_{0}}{2 \pi \beta c h e V_{\mathrm{rf}}\left|\sin \phi_{s}\right|}\right)+\frac{3}{\gamma \beta^{2}} \frac{d \gamma}{d s} \frac{d \tau}{d s}=0
$$

where $d \gamma / d s$ is the gradient of acceleration, which we assume to be a constant, and the synchrotron tune is

$$
\nu_{s}=\sqrt{\frac{h M e V_{\mathrm{rf}}\left|\sin \phi_{s}\right|}{2 \pi \beta^{2} \gamma^{3} E_{0}}} .
$$

In the above, we have introduced the $\mathrm{rf}$ harmonic $h=\omega_{\mathrm{rf}} / \omega_{0}$, where $\omega_{0} / 2 \pi=v / L_{0}$ is the number of linac period a beam particle travels per unit time. The quantity inside the brackets of Eq. (7.34) can be considered as a shift of the arrival time

$$
\tau \rightarrow \bar{\tau}=\tau-\frac{v \Delta p_{n}^{(0)} L_{0}}{2 \pi \beta \operatorname{che} V_{\mathrm{rf}}\left|\sin \phi_{s}\right|} .
$$

We notice that the energy delivered by the impulse $v \Delta p_{n}^{(0)}$ is velocity independent [see Eq. (7.39)]. Thus the shift in arrival time depends on time only through the beam velocity $\beta c$ in the denominator, and can be considered as weak. Ignoring this weak dependency, the equation of motion can be simplified to

$$
\frac{d^{2} \bar{\tau}}{d s^{2}}+\frac{4 \pi^{2} \nu_{s}^{2}}{L_{0}^{2}} \bar{\tau}+\frac{3}{\gamma \beta^{2}} \frac{d \gamma}{d s} \frac{d \bar{\tau}}{d s}=0 .
$$

The first two terms depict synchrotron oscillation, while the third term denotes adiabatic damping as a result of acceleration. Adiabatic damping will damp synchrotron oscillation, 
resulting in more stable bunches in the longitudinal phase space. For this reason, adiabatic damping can be first ignored in a preliminary investigation of beam stability, and its inclusion is only required when the preliminary investigation arrives at an unstable result.

When the adiabatic damping term in Eq. (7.37) is omitted, it is easy to conclude that the longitudinal HOM effect on the bunch position is small provided that the shift of the bunch center is very much less than the rms bunch length $\sigma_{t}$. A shift parameter $\Upsilon^{(0)}$ can be introduced and we require

$$
\Upsilon^{(0)}=\left|\frac{v \Delta p_{n}^{(0)} L_{0}}{2 \pi \beta c \sigma_{t} h e V_{\text {rf }} \sin \phi_{s}}\right| \ll 1 .
$$

Otherwise there will be a bunch length increase and synchrotron oscillation will become unstable. Notice that $\Upsilon^{(0)}$ depends only rather weakly on the beam energy, especially near the end of the linac.

Now after passage of an infinite number of bunches, the energy imparted on a beam particle from the excitation of the $n^{\text {th }}$ monopole HOM is, for one cavity,

$$
\begin{aligned}
v \Delta p_{n}^{(0)} & =e^{2} N_{b} \omega_{n}^{(0)}\left(\frac{R_{n}}{Q_{n}}\right)^{(0)} \sum_{k=0}^{\infty} e^{-k \omega_{n}^{(0)} T_{b} / 2 Q_{n L}^{(0)}} \cos k \omega_{n}^{(0)} T_{b} \\
& =e^{2} N_{b} \omega_{n}^{(0)} \mathcal{R} e \frac{\left(R_{n} / Q_{n}\right)^{(0)}}{1-\exp \left(-\omega_{n}^{(0)} T_{b} / 2 Q_{n L}^{(0)}+i \omega_{n}^{(0)} T_{b}\right)} \\
& =\frac{e^{2} N_{b} \omega_{n}^{(0)}\left(R_{n} / Q_{n}\right)^{(0)}\left(1-e^{-\theta} \cos \phi\right)}{1-2 e^{-\theta} \cos \phi+e^{-2 \theta}},
\end{aligned}
$$

where $\theta=\omega_{n}^{(0)} T_{b} / 2 Q_{n L}^{(0)}$ and $\phi=\omega_{n}^{(0)} T_{b}$. For the Ellip082 section, there are $M=5$ cavities in each period, and the synchrotron phase is $\phi_{s}=-10^{\circ}$. The average energy gain per period is $\Delta E=59.6 \mathrm{MeV}$ or $d \gamma / d s=6.74 \times 10^{-3} \mathrm{~m}^{-1}$. The mean rf voltage per cavity is $V_{\mathrm{rf}}=\Delta E /\left(e M \cos \phi_{s}\right)=12.1 \mathrm{MV}$. The period length is $L_{0}=9.62 \mathrm{~m}$. At the mean velocity of $\beta_{G}=0.82$, the mean period frequency is $\omega_{0} / 2 \pi=v / L_{0}=25.6 \mathrm{MHz}$. The rf harmonic is therefore $h=\omega_{\mathrm{rf}} / \omega_{0}=25.44$. The rf harmonic $h$ of a circular accelerator must be an integer because a particle is seeing the same rf cavity every revolution turn and the rf voltage wave must be in phase with the particle in passage. In a linac, however, the particle is seeing different rf cavities from period to period. The phases of the rf wave of the rf cavities in successive periods can always be adjusted independently to deliver the correct energy to the particle. For this reason, there is no need for the rf harmonic to be an integer. At the entrance of the Ellip082 section, the relativistic factor of the beam particle is $\gamma=1.3949$ and $\beta=0.6969$. 


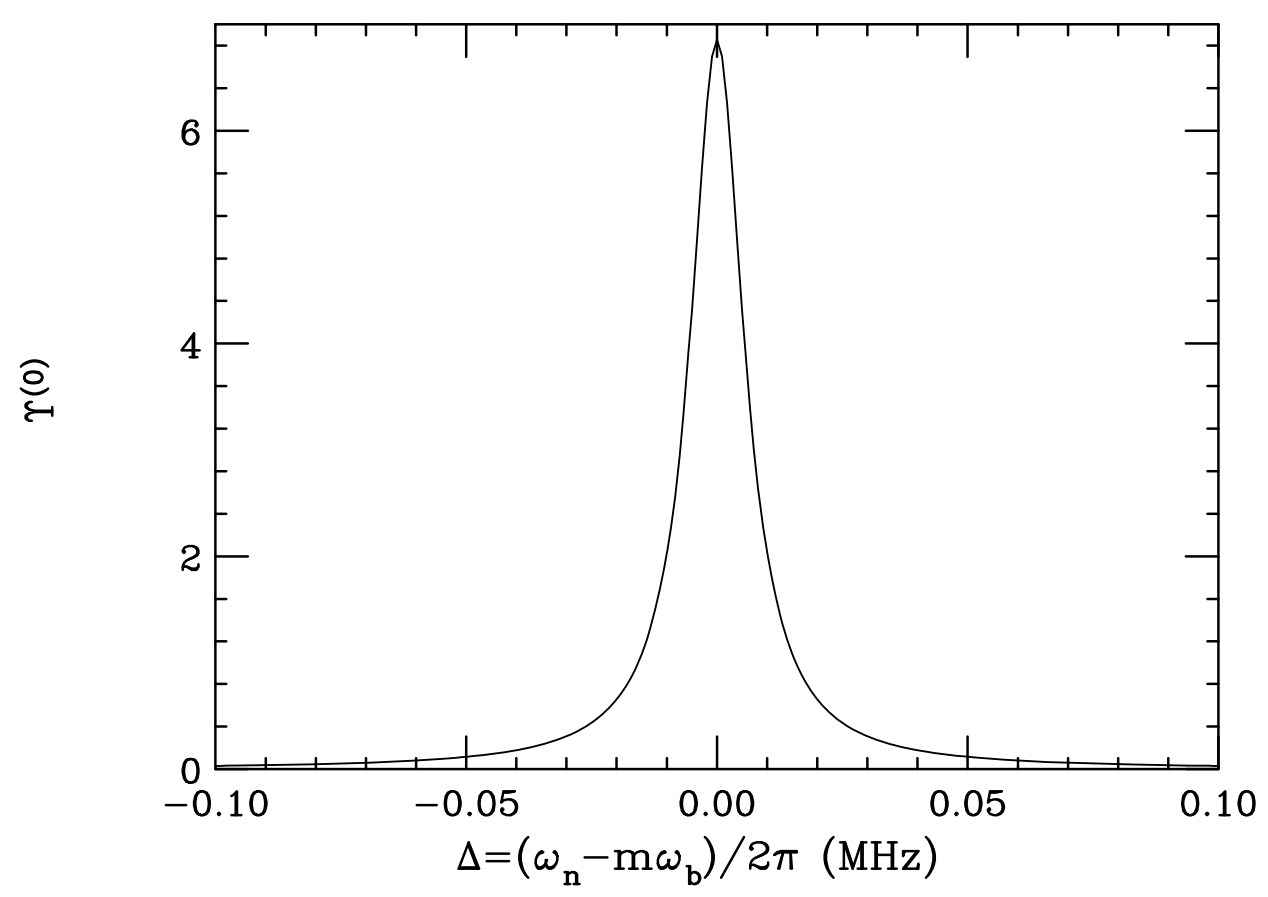

Figure 11: The shift parameter $\Upsilon^{(0)}$ as a function of deviation of the monopole HOM frequency from a multiple of the beam frequency. The parameter shows the shift of the bunch center in the longitudinal direction as compared with the rms bunch width.

When the monopole HOM is of frequency exactly a multiple of the beam frequency, $\cos \phi=1$ and the HOM voltage induced by each successive bunch adds together in phase. The accumulation is finite only because of the finite external quality factor $Q_{n L}^{(0)}$. The total accumulated HOM voltage deposited on a beam particle becomes

$$
v \Delta p_{n}^{(0)} \rightarrow 2 I_{b}\left(\frac{R_{n}}{Q_{n}}\right)^{(0)} Q_{n L}^{(0)} .
$$

For the most offending monopole HOM at $1241 \mathrm{MHz}$ and $\left(R_{n} / Q_{n}\right)^{(0)}=137 \Omega$, Fig. 11 shows that the shift parameter $\Upsilon^{(0)}$ of Eq. (7.38) is roughly $\sim 7$, where the rms bunch length of $\sigma_{t} \sim 5.2 \mathrm{ps}$ has been used. However, the shift parameter drops by three orders of magnitude if the HOM frequency differs from the multiple of the beam frequency by $1 \mathrm{MHz}$. In other words, there is no concern at all.

Because of the slip parameter in a linac is proportional to $\gamma^{-2}$, the synchrotron tune $\nu_{s}$ in Eq. (7.37) is strongly energy-dependent and therefore time-dependent. It decreases as the beam particle transverses the linac, from $\nu_{s}=0.083$ at the entrance of the Ellip082 section to $\nu_{s}=0.024$ at exit. Although adiabatic damping is expected from the third term 
in Eq. (7.34), to understand the energy-dependent effect in more detail and correctly, it is necessary to solve Eq. (7.37) more exactly. A numerical solution is recommended.

\section{Comments}

Here, we have made a few estimates, but far from complete, about the effects of the HOMs in the elliptical SRF cavities of the C-ADS linac. We just list a few comments:

1. Although a geometric parameter, $R / Q$ of a cavity depends on the velocity of the beam particle passing through it. Here, what we list in Figs. 5 and 8 are only the maximum $R / Q$ for some beam particle velocity nearby. The implication is that the HOM effects can be different when the actually velocity dependency of $R / Q$ is properly considered.

2. We have given some very rough estimates concerning the effects of HOMs of the elliptical SRF cavities. We discover that the effects are big only when the HOM frequencies are nearly exact multiples of the beam frequency, and the effects diminish drastically when the HOM frequencies are far away from the beam frequency multiples. For the C$\mathrm{ADS}$, there is only one linac operation, which is CW. As a results, the beam frequency spectral lines are well separated by $162.5 \mathrm{MHz}$. The possibility that a HOM frequency is extremely near a beam frequency multiple is rather remote. Even if this happens accidentally, the offending HOM frequency can be detuned by deforming the cavity appropriately.

3. A more detailed examination of HOM issues requires actual tracking of a C-ADS beam through the Ellip063 and Ellip082 sections with an operational lattice for these sections.

4. By definition, monopole modes imply that the field viewed in the cross-section of a cavity is independent of angle. For example, the HOM near $1600 \mathrm{MHz}$, shown in Fig. 12 is a monopole mode. The center picture shows that electric field is radially outside at the opening of a cell in the cavity. The longitudinal electric field is nearly zero there. However, in between cells, the longitudinal electric field is nonzero and this gives rise to the $R / Q$ integration along the axis of the cavity [see Eq. (5.21)]. Unlike the fundamental monopole mode, here for a particle passes off-center, it sees a transverse force and will be deflected inwards or outwards radially. If the particle is deflected inwards at entrance and outwards at exit, the net deflection resembles an alternating focusing and defocusing quadrupole. On the other hand, if the particle is 


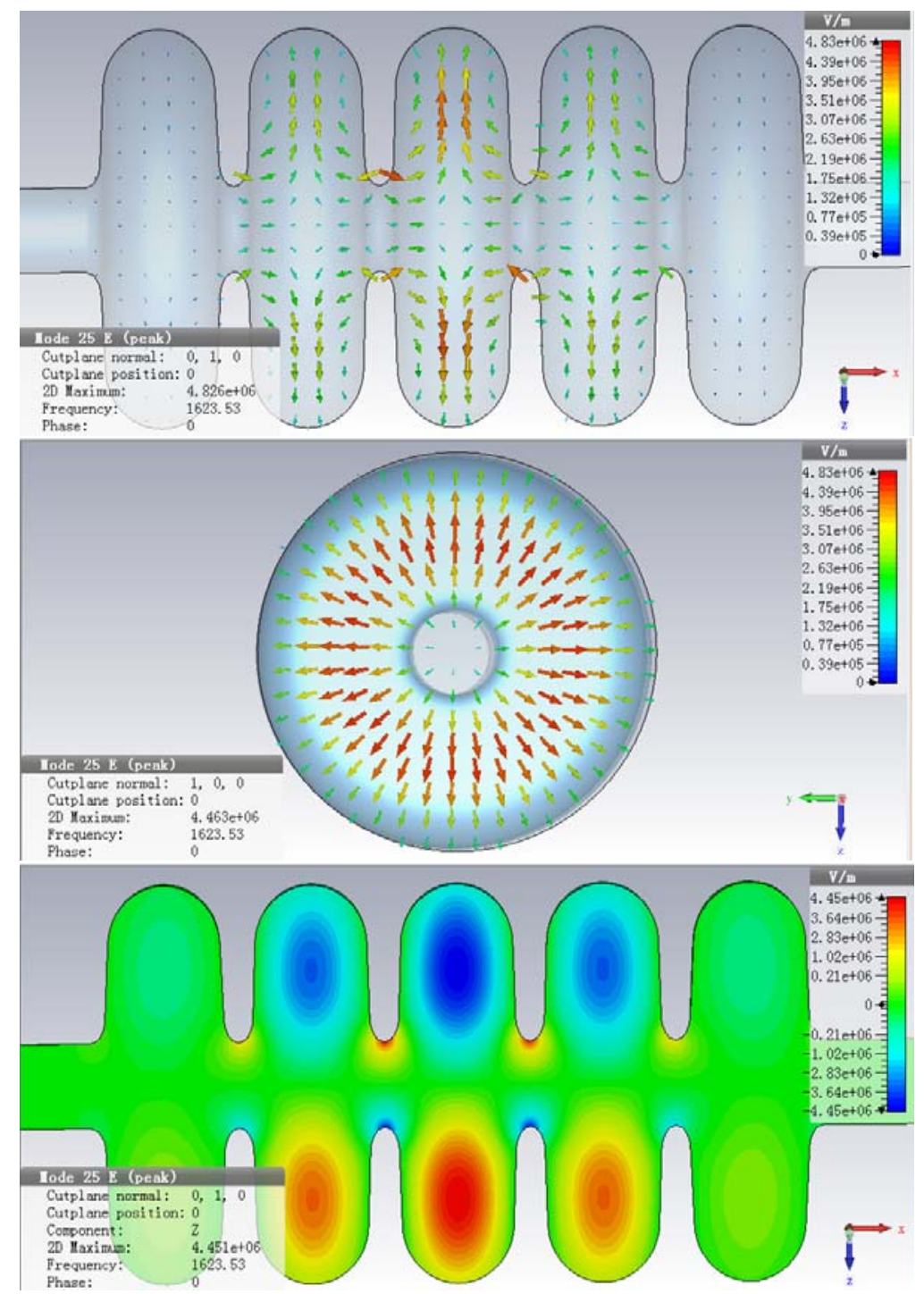

Figure 12: Electric field distribution in a monopole HOM near $1600 \mathrm{MHz}$. Top: 3D electric field viewed from the direction perpendicular to the cavity axis. Middle: the $3 \mathrm{D}$ electric field viewed from the direction along the cavity axis. Bottom: the 2D electric field viewed on the cutting plane along the cavity axis. Note that the color codes in the three plots are different. 
deflected inwards (or outwards) both at entrance and exit, the deflection will accumulate resulting in beam loss. Transverse effects should therefore be analyzed as well. This constitutes one of the many issues further investigation should address.

\section{References}

[1] Conceptual Physics Design on the C-ADS Accelerators, Ed. Jingyu Tang and Zhihui Li, Institute of High Energy Physics, CAS and Institute of Modern Physics, CAS, August 2012, IHEP-CADS-Report/2012-01E.

[2] V. Yakovlev, M. Champion, I. Gonin, T. Khabbiboulline, A. Lunin, A. Saini, N. Solyak, A. Sukhanov, and A. Vostrikov, HOM Couplers for Project X: Are they needed?, Project X Collaboration Meeting, Spallation Neutron Source facility at Oak Ridge National Laboratory, April 12-14, 2011.

[3] A. Sukhanov, A. Lunin, V. Yakovlev, M. Awida, M. Champion, C. Ginsburg, I. Gonin, C. Grimm, T. Khabbiboulline, T. Nicol, Yu. Orlov, A. Saini, D. Sergatskov, N. Solyak, and A. Vostrikov, Higher order modes in Project-X linac, Nucl. Inst. Meth. in Physics Research A734, 9, (2014)

[4] The normalized longitudinal emittance is uniquely defined as $\epsilon_{z \Delta p}^{N}=\sigma_{z} \sigma_{\Delta p}$. However, there are many ways to express the unnormalized emittance, like $\epsilon_{z z^{\prime}}=\sigma_{z} \sigma_{z^{\prime}}$ with $z^{\prime}=\Delta \beta / \beta$ in the length-relative-velocity unit, $\epsilon_{z, \Delta p / p}=\sigma_{z} \sigma_{\Delta p / p}$ in the length-relativemomentum-offset unit, $\epsilon_{W z}=\sigma_{W} \sigma_{z}$ in the energy-length unit, and $\epsilon_{W t}=\sigma_{W} \sigma_{t}$ in the energy-time unit. Since $\Delta \gamma=\beta \gamma^{3} \Delta \beta$, we have $\epsilon_{W z}=\beta^{2} \gamma^{3} E_{0} \epsilon_{z z^{\prime}}$. Since $\Delta \beta \gamma /(\beta \gamma)=$ $\Delta \gamma /\left(\beta^{2} \gamma\right)=\gamma^{2} \Delta \beta / \beta$, we have $\epsilon_{z \Delta p / p}=\gamma^{2} \epsilon_{z z^{\prime}}$ as well as $\epsilon_{z \Delta p}^{N}=\beta \gamma \epsilon_{z \Delta p / p}=\beta \gamma^{3} E_{0} \epsilon_{z z^{\prime}}$, where $E_{0}$ is the particle rest mass. Note that some authors may include a factor of $\pi$ in the emittance definitions. However, a common convention is to have it emitted, but have it included in the unit of the emittance instead.

[5] https://www.cst.com

[6] http://irfu.cea.fr/Sacm/en/logiciels/index3.php

[7] Here $\sigma_{U_{n}}$ is the rms value and $\tilde{U}_{n}^{(0)}$ is the peak value. The $\sqrt{2}$ is the factor used in [3]. We consider this factor arbitrary. If one likes one can use the factor 2 instead.

[8] Reference 1, p.80-82.

[9] A.W. Chao, Physics of Collective Beam Instabilities in High Energy Accelerators, Wiley 1993. 